\title{
Anti-Insect Activities of Plant Extracts on the Diamondback Moth, Plutella xylostella (L.)
}

\author{
R. Sangavi* and Y.S. Johnson Thangaraj Edward
}

Department of Agricultural Entomology, Agricultural College and Research Institute, Tamil Nadu Agricultural University, Coimbatore - 641 003, Tamil Nadu, India

*Corresponding author

\begin{abstract}
Keywords
Plant extracts, Diamondback moth, Larval mortality, Oviposition deterrence, Antifeedant activity.

Article Info

Accepted: 04 October 2017 Available Online: 10 December 2017 ingredients (Shelton et al., 2008); and this has triggered the development of alternative measures, including botanical insecticides (Oyedokun et al., 2011). Experiments were conducted under laboratory conditions to study the anti-insect activities of locally available plants against diamondback moth, Plutella xylostella (L.) (Lepidoptera: Plutellidae). In the preliminary screening of 45 plants, higher per cent mortality was recorded on 5 DAT in the aqueous leaf extract (10\%) of Prosopis juliflora (96.67\%), Andrographis paniculata (80.00 \%), Sesbania grandiflora (73.33\%), Annona squamosa (66.67 \%), Jatropha curcas (66.67 \%), Abutilon indicum (60.00\%), Curcuma longa (56.67\%) and Azadirachta indica (53.33\%). The highest pupal mortality was recorded in Cymbopogon schoenanthus $(60.00$ $\%)$, Gliricidia sepium (50.00\%), Cyperus rotundus (53.33\%), Azadirachta indica (46.67 $\%)$ and Tagetes tenuifolia (30.00\%). No adult emergence was observed in P. juliflora, A. paniculata and $A$. indica. The effect of the selected five plants viz., P. juliflora, A. paniculata, S. grandiflora, J. curcas and C. longa on the growth and development of $P$. xylostella was confirmed in the confirmative experiment. The highest anti- feedant activity against $P$. xylostella was observed in P. juliflora $(58.41 \%)$. The oviposition deterrence percentage by free choice test was found to be the highest in S. grandiflora $(85.95 \%)$ and by no choice test was found to be the highest in P. juliflora (61.26\%).
\end{abstract}

\section{A B S T R A C T}

The diamondback moth is known globally by many as the most destructive and economically important insect pest of cruciferous crops. It is also known to have developed resistance to numerous synthetic insecticides including those with newer active

\section{Introduction}

Cruciferous vegetables are the important dietary source of vitamins (A, B1 and C) and minerals like phosphorus, potassium, sodium, calcium and iron which are essentially needed as supplement in human diet. The varied agro-climatic conditions prevailing in India are able to produce variety of vegetables throughout the year. India is the second largest producer of cruciferous vegetables in the world. It is grown over an area of 3.12 million ha in the world and 0.331 million ha in India. However, the average productivity of cabbage in India is about 22.0 metric tonnes/ha which is less than World's productivity of 22.3 million tonnes/ha (NHB, 2017). Many species of insects attack the commonly growing cruciferous vegetables like cabbage (Brassica oleracea L. var. capitata), cauliflower (Brassica oleracea L. var. botrytis), turnip (Brassica rapa L.), carrot 
(Daccus carota L.) and mustard (Brassica campestris L. var. toria and Brassica campestris L. var. sarson). The crop is prone for infestation by a number of insect pests consisting of sucking and defoliating insects starting from germination to harvesting stage of the crop.

Among several species of insects, diamondback moth (DBM), Plutella xylostella (L.), Lepidoptera: Plutellidae is the most revenging pest identified by Fletcher on cruciferous vegetables (Fletcher, 1914). In India, a total of 37 insect pests have been reported to feed on cabbage, of which the DBM causes the loss of about 35 per cent with intensive control measures (Mohan and Gujar, 2003).

Estimated total costs associated with damage and management of DBM worldwide was 4-5 billion US dollers per annum (Zalucki et al., 2012) and in India, the estimated cost for the control of DBM is about 16 million US\$ per annum (Sharma and Singh, 2014).

Utilization of conventional synthetic insecticides posed certain problems such as adverse effects on natural enemies, development of resistance in target pests and pest resurgence. Hazardous implications of these pesticides and their residue at various trophic levels have also caused incalculable damage to every aspect of environment, globally.

In India, the first report of $P$. xylostella resistance to insecticides (DDT and parathion) was made by Verma and Sandhu (1968) in Ludhiana (Punjab). Subsequently this was confirmed by Deshmukh and Saramma (1973), to fenitrothion and malathion (Chawla and Kalra, 1976); cypermethrin, decamethrin and quinalphos (Saxena et al., 1989) and quinalphos (Chawla and Joia, 1991). Some population of $P$. xylostella developed resistance to new generation insecticides such as spinosad, avermectins, indoxacarb, emamectin benzoate and Bacillus thuringiensis, B.t. Cry toxins in the field (Sayyed and Wright, 2006; Zhao et al., 2006; Li et al., 2007 and Pu et al., 2010).

Currently, eco-friendly agriculture and demand for organic foods are gaining worldwide attention due to drawbacks associated with the agricultural chemicals. Plant products have been suggested as alternative sources for insect control products because some are selective, biodegrade to nontoxic products, and have few effects on non-target organisms and the environment (Singh et al., 2006; Isman, 2008).

\section{Materials and Methods}

Studies were carried out to evaluate the antiinsect effects of different plant extracts on the diamondback moth, Plutella xylostella (L.) at the Department of Agricultural Entomology, Agricultural College and Research Institute, Coimbatore during 2016 - 2017.

The different plant parts from 45 plants viz., leaves, flowers, fruit and roots were collected, weighed and shade dried for a week again dry weight was recorded and then powdered. The powdered plant products were again fine powdered by pulveriser and sieved to a size of $0.5 \mathrm{~mm}$.

\section{Bioassay}

The leaf dipping bioassay method described by Tabashnik and Cushing (1987) was adopted to evaluate the insecticidal action of 45 botanicals against $P$. xylostella larvae. Cabbage leaves were washed with distilled water and dried for about 10 minutes. Aqueous extract of different botanicals were tested at 10 per cent concentration along with NSKE 5 per cent as standard check. 
Completely untreated leaves were used as control.

Fresh cabbage leaf discs $(7.5 \mathrm{~cm}$ diameter) were cut from fully expanded cabbage leaves. The discs were dipped for 60 seconds in the test solutions.

These treated and air dried leaves were placed in a petriplates lined with moist filter paper. In each petriplate 10 third instar larvae was released using a camel hair brush and allowed to feed for 48 hours. After 48 hours, treated leaves were removed and fresh untreated leaves were given.

Three replications of each of the treatment with 10 larvae per replicate were maintained. Readings were taken at 24 hours intervals up to adult emergence for larval mortality, pupal mortality and adult emergence. Any malformation in the treatments was also observed.

Mortality $(\%)=\frac{\text { Number of insects died }}{\text { Total number of insects released }} \times 100$

From the 45 botanicals tested, the best 5 botanicals were selected, tested for Antifeedant and Oviposition deterrent activity.

Assessment of anti-feedant activity of selected botanicals on $P$. xylostella

Progressive consumption of leaf area was measured both in control and in treatments 24 and 48 hours after release of larvae using graphical method. Three replications of each of the treatments with 10 larvae per replicate were maintained.

Leaf area consumed in control Leaf area consumed in treatment Anti- feedant activity $(\%)=$ X100

Leaf area consumed in control
Evaluation of oviposition deterrent action of selected botanicals on $P$. xylostella

\section{Free choice test}

In choice test, mustard seedlings, were sprayed with selected aqueous extracts of botanicals at 10 per cent concentration using a hand atomizer. Once treated, plants were transferred immediately to oviposition cages and placed in a random order such that each cage consisted of a set of plants with different treatments arranged in different placement including the control. Three replications were maintained and 14 pairs of adults were released into each cage at the rate of 2 pairs per plant. Sugar solution $(10 \%)$ was provided for adult moth feeding. The number of eggs laid was recorded after 48 hours and oviposition deterrence was calculated with the formula of Villalobos and Robledo, (1998).

Oviposition deterrence $(\%)=100 \times(1-$ $\mathrm{NEt} / \mathrm{NEc}$ )

Where, NEt is the number of eggs in treatment and NEc is the number of eggs in control.

\section{No-choice test}

For each treatment a separate cage was used. Untreated mustard seedlings kept in a separate cage were treated as control. The experiment was replicated thrice. Two pairs of one day old adults of $P$. xylostella were released inside the cage for oviposition.

\section{Results and Discussion}

\section{Effect on larval mortality, pupal mortality and adult emergence}

Among the thirteen aqueous extract (10\%) of plants belonging to the families, Acanthaceae, Acoraceae, Amaranthaceae, Anacardiaceae, 
Annonaceae, Apiaceae, Apocynaceae, Araceae and Asteraceae, Andrographis paniculata recorded the maximum larval mortality (46.67\%), followed by Artemisia pallens (20.00\%), Achyranthes aspera (16.67 $\%)$, Annona squamosa (13.33 \%) and no mortality was observed in Colocasia esculenta, Tagetes tenuifolia flower and Tridax procumbens one day after treatment (DAT).

Highest per cent mortality was recorded on 5 DAT in A. paniculata (80.00\%) followed by Annona squamosa $(66.67 \%)$ which were significantly higher than all other plant products tested including the check, neem seed kernel extract (NSKE) at 5 per cent.

The highest pupal mortality was recorded in Tagetes tenuifolia leaf $(30.00 \%)$ followed by Achyranthes aspera, Acorus calamus and T. tenuifolia flower $(26.67 \%)$. None of the larvae reached the adult stage in Andrographis paniculata. Malformed adults (Plate 5) emergence was observed in Centella asiatica $(6.67 \%)$ followed by Allamanda catharitica, Catharanthus roseus, $T$. tenuifolia and A. calamus (3.33\%) (Table 1).

Among the nine aqueous extract $(10 \%)$ of plants belonging to the family Fabaceae, on 1 DAT highest larval mortality was recorded on Prosopis juliflora $(53.33 \%)$ and no mortality was observed in Cassia alata, Cassia fistula, Delonix regia, Gliricidia sepium and Senna auriculata.

On 2 DAT, Sesbania grandiflora was on statistically par with $P$. juliflora. Highest mortality recorded on 5 DAT was in $P$. juliflora $(96.67 \%)$ which was significantly higher and was followed by $S$. grandiflora $(73.33 \%)$.The check NSKE (5\%) recorded 33.33 per cent mortality. The highest pupal mortality was recorded in G. sepium $(50.00 \%)$. No adult emergence was recorded in $P$. juliflora. The malformed adult was observed in D. regia (10.00\%) and Cassia fistula $(3.33 \%)$ (Table 2).

Among the twelve aqueous extract $(10 \%)$ of plants belonging to the families, Bignoniaceae, Cyperaceae, Euphorbiaceae, Lamiaceae, Malvaceae, Meliaceae, Myrtaceae, Pedaliaceae, Phyllanthaceae and Piperaceae, maximum larval mortality of 40.00 per cent was recorded in Abutilon indicum and Phylanthus niruri and no mortality was observed in Cyperus rotundus, Pedalium murex and NSKE (5\%) at 1 DAT. Highest mortality recorded on 5 DAT was in Jatropha curcas $(66.67 \%)$ which was significantly higher followed by Abutilon indicum $(60.00 \%)$ and Azadirachta indica $(53.33 \%)$.The pupal mortality was highest in C. rotundus $(53.33 \%)$ which was statistically on par with $A$. indica (46.67 \%). The malformed adult was observed in $M$. azedarach $(10.00 \%), \quad$ S. campanulata (6.67\%) and $O$. tenuiflorum (3.33\%) (Table $3)$.

Among the eleven aqueous extract $(10 \%)$ of plants belonging to the families, Poaceae, Polygonaceae, Rutaceae, Sapindaceae, Solanaceae, Sterculiaceae, Verbenaceae and Zingiberaceae, on 1 DAT maximum larval mortality was recorded on Murraya koenigii (20.00\%). On 3 DAT, mortality was highest in M. koenigii and Solanum virginianum of (26.67 and $20.00 \%$ ) which is statistically on par with the check, NSKE at 5 per cent $(20.00$ $\%)$. Significantly higher mortality was recorded in Curcuma longa (56.67\%) on 5 DAT. The pupal mortality was highest in $C$. schoenanthus $(60.00 \%)$. The lowest adult emergence was recorded in C. longa (13.33 $\%)$ and the highest in Cardiospermum halicacabum $(80.00 \%)$. The malformed adult was observed in A. muricatus, $C$. schoenanthus and A. leptopus (6.67\%) (Table 4). 
Table.1 Effect of aqueous plant extracts (10\%) belonging to the Family, Acanthaceae, Acoraceae, Amaranthaceae, Anacardiaceae, Annonaceae, Apiaceae, Apocynaceae, Araceae and Asteraceae on the growth and development of the diamondback moth, P. xylostella

\begin{tabular}{|c|c|c|c|c|c|c|c|c|}
\hline \multirow[b]{2}{*}{ Treatments } & \multicolumn{5}{|c|}{ Larval mortality (\%) } & \multirow{2}{*}{$\begin{array}{c}\text { Pupal } \\
\text { Mortaliy } \\
(\%) \\
\end{array}$} & \multicolumn{2}{|c|}{ Adult emergence (\%) } \\
\hline & 1DAT & 2DAT & 3DAT & 4DAT & 5DAT & & Normal & Malformed \\
\hline $\begin{array}{l}\text { Andrographis } \\
\text { paniculata(leaf) }\end{array}$ & $\begin{array}{c}46.67 \\
(43.07)^{\mathrm{a}}\end{array}$ & $\begin{array}{c}73.33 \\
(58.89)^{\mathrm{a}}\end{array}$ & $\begin{array}{c}73.33 \\
(59.21)^{\mathrm{a}}\end{array}$ & $\begin{array}{c}80.00 \\
(63.77)^{\mathrm{a}}\end{array}$ & $\begin{array}{c}80.00 \\
(63.77)^{\mathrm{a}}\end{array}$ & $\begin{array}{c}20.00 \\
(26.55)^{\mathrm{b}}\end{array}$ & $\begin{array}{c}0.00 \\
(4.05)^{\mathrm{g}}\end{array}$ & $\begin{array}{c}0.00 \\
(4.05)^{\mathrm{c}}\end{array}$ \\
\hline $\begin{array}{l}\text { Acorus calamus } \\
\text { (rhizome) }\end{array}$ & $\begin{array}{c}3.33 \\
(10.52)^{\mathrm{g}}\end{array}$ & $\begin{array}{c}16.67 \\
(24.09)^{\mathrm{c}}\end{array}$ & $\begin{array}{c}20.00 \\
(26.91)^{\mathrm{e}}\end{array}$ & $\begin{array}{c}30.00 \\
(33.51)^{\mathrm{e}}\end{array}$ & $\begin{array}{c}30.00 \\
(33.51)^{\mathrm{c}}\end{array}$ & $\begin{array}{c}26.67 \\
(31.40)^{\mathrm{a}}\end{array}$ & $\begin{array}{c}40.00 \\
(39.51)^{\mathrm{e}}\end{array}$ & $\begin{array}{c}3.33 \\
(11.29)^{\mathrm{b}}\end{array}$ \\
\hline $\begin{array}{l}\text { Achyranthes aspera } \\
\text { (leaf) }\end{array}$ & $\begin{array}{c}16.67 \\
(24.09)^{\mathrm{c}}\end{array}$ & $\begin{array}{c}23.33 \\
(28.87)^{\mathrm{b}}\end{array}$ & $\begin{array}{c}26.67 \\
(31.40)^{\mathrm{d}}\end{array}$ & $\begin{array}{c}33.33 \\
(35.55)^{\mathrm{d}}\end{array}$ & $\begin{array}{c}33.33 \\
(35.55)^{\mathrm{c}}\end{array}$ & $\begin{array}{c}26.67 \\
(31.40)^{\mathrm{a}}\end{array}$ & $\begin{array}{c}40.00 \\
(39.51)^{\mathrm{e}}\end{array}$ & $\begin{array}{c}0.00 \\
(4.05)^{\mathrm{c}}\end{array}$ \\
\hline $\begin{array}{l}\text { Anacardium occidentale } \\
\text { (leaf) }\end{array}$ & $\begin{array}{c}6.67 \\
(14.96)^{\mathrm{f}}\end{array}$ & $\begin{array}{c}6.67 \\
(14.96)^{\mathrm{e}}\end{array}$ & $\begin{array}{c}6.67 \\
(15.52)^{\mathrm{h}}\end{array}$ & $\begin{array}{c}13.33 \\
(21.83)^{\mathrm{f}}\end{array}$ & $\begin{array}{c}13.33 \\
(21.83)^{\mathrm{e}}\end{array}$ & $\begin{array}{l}20.00 \\
(26.91)^{\mathrm{b}}\end{array}$ & $\begin{array}{c}66.67 \\
(55.02)^{\mathrm{d}}\end{array}$ & $\begin{array}{c}0.00 \\
(4.05)^{\mathrm{c}}\end{array}$ \\
\hline $\begin{array}{l}\text { Annona squamos } \\
\text { (leaf) }\end{array}$ & $\begin{array}{c}13.33 \\
(21.41)^{\mathrm{d}}\end{array}$ & $\begin{array}{c}26.67 \\
(31.08)^{\mathrm{b}}\end{array}$ & $\begin{array}{c}40.00 \\
(39.51)^{\mathrm{c}}\end{array}$ & $\begin{array}{c}20.00 \\
(26.91)^{\mathrm{e}}\end{array}$ & $\begin{array}{c}66.70 \\
(47.18)^{\mathrm{b}}\end{array}$ & $\begin{array}{c}0.00 \\
(4.05)^{\mathrm{f}}\end{array}$ & $\begin{array}{c}46.66 \\
(43.36)^{\mathrm{e}}\end{array}$ & $\begin{array}{c}0.00 \\
(4.05)^{\mathrm{c}}\end{array}$ \\
\hline $\begin{array}{l}\text { Centella asiatica } \\
\text { (leaf) }\end{array}$ & $\begin{array}{c}3.33 \\
(10.52)^{g}\end{array}$ & $\begin{array}{c}3.33 \\
(10.52)^{\mathrm{f}}\end{array}$ & $\begin{array}{c}3.33 \\
(11.29)^{\mathrm{i}}\end{array}$ & $\begin{array}{c}3.33 \\
(11.29)^{\mathrm{g}}\end{array}$ & $\begin{array}{c}3.33 \\
(11.29)^{\mathrm{f}}\end{array}$ & $\begin{array}{c}16.67 \\
(24.47)^{\mathrm{b}}\end{array}$ & $\begin{array}{c}73.33 \\
(59.21)^{\mathrm{c}}\end{array}$ & $\begin{array}{c}6.67 \\
(15.52)^{\mathrm{a}}\end{array}$ \\
\hline $\begin{array}{l}\text { Allamanda catharitica } \\
\text { (leaf) }\end{array}$ & $\begin{array}{c}10.00 \\
(18.43)^{\mathrm{e}}\end{array}$ & $\begin{array}{c}16.67 \\
(24.09)^{\mathrm{c}}\end{array}$ & $\begin{array}{c}16.67 \\
(24.47)^{\mathrm{ef}}\end{array}$ & $\begin{array}{c}20.00 \\
(26.91)^{\mathrm{e}}\end{array}$ & $\begin{array}{c}20.00 \\
(26.91)^{\mathrm{d}}\end{array}$ & $\begin{array}{c}13.33 \\
(21.83)^{\mathrm{c}}\end{array}$ & $\begin{array}{c}63.33 \\
(53.01)^{\mathrm{cd}}\end{array}$ & $\begin{array}{c}3.33 \\
(11.29)^{\mathrm{b}}\end{array}$ \\
\hline $\begin{array}{l}\text { Catharanthus roseus } \\
\text { (leaf) }\end{array}$ & $\begin{array}{c}3.33 \\
(10.52)^{\mathrm{g}}\end{array}$ & $\begin{array}{c}10.00 \\
(18.43)^{\mathrm{e}}\end{array}$ & $\begin{array}{c}13.33 \\
(21.83)^{\mathrm{fg}}\end{array}$ & $\begin{array}{c}13.33 \\
(21.83)^{\mathrm{f}}\end{array}$ & $\begin{array}{c}13.33 \\
(21.83)^{\mathrm{e}}\end{array}$ & $\begin{array}{c}6.67 \\
(15.52)^{\mathrm{e}}\end{array}$ & $\begin{array}{c}76.67 \\
(61.43)^{\mathrm{b}}\end{array}$ & $\begin{array}{c}3.33 \\
(11.29)^{\mathrm{b}}\end{array}$ \\
\hline $\begin{array}{l}\text { Colocasia esculenta } \\
\text { (leaf) }\end{array}$ & $\begin{array}{c}0.00 \\
(4.05)^{\mathrm{h}}\end{array}$ & $\begin{array}{c}13.33 \\
(21.41)^{\mathrm{d}}\end{array}$ & $\begin{array}{c}13.33 \\
(21.83)^{\mathrm{fg}}\end{array}$ & $\begin{array}{c}40.00 \\
(39.51)^{\mathrm{c}}\end{array}$ & $\begin{array}{c}53.33 \\
(47.18)^{\mathrm{b}}\end{array}$ & $\begin{array}{c}20.00 \\
(26.91)^{\mathrm{b}}\end{array}$ & $\begin{array}{c}26.67 \\
(31.40)^{\mathrm{f}}\end{array}$ & $\begin{array}{c}0.00 \\
(4.05)^{\mathrm{c}}\end{array}$ \\
\hline $\begin{array}{l}\text { Artemisia pallens } \\
\text { (leaf) }\end{array}$ & $\begin{array}{c}20.00 \\
(26.55)^{\mathrm{b}}\end{array}$ & $\begin{array}{c}10.00 \\
(18.43)^{\mathrm{d}}\end{array}$ & $\begin{array}{c}26.67 \\
(31.40)^{\mathrm{d}}\end{array}$ & $\begin{array}{c}26.67 \\
(31.40)^{\mathrm{de}}\end{array}$ & $\begin{array}{c}30.00 \\
(33.51)^{\mathrm{c}}\end{array}$ & $\begin{array}{c}10.00 \\
(18.90)^{\mathrm{d}}\end{array}$ & $\begin{array}{c}60.00 \\
(51.04)^{\mathrm{d}}\end{array}$ & $\begin{array}{c}0.00 \\
(4.05)^{\mathrm{c}}\end{array}$ \\
\hline $\begin{array}{l}\text { Tagetes tenuifolia } \\
\text { (leaf) }\end{array}$ & $\begin{array}{c}0.00 \\
(4.05)^{\mathrm{h}}\end{array}$ & $\begin{array}{c}6.67 \\
(14.96)^{\mathrm{e}}\end{array}$ & $\begin{array}{c}3.33 \\
(11.29)^{\mathrm{i}}\end{array}$ & $\begin{array}{c}20.00 \\
(26.91)^{\mathrm{e}}\end{array}$ & $\begin{array}{c}20.00 \\
(26.91)^{\mathrm{d}}\end{array}$ & $\begin{array}{c}30.00 \\
(33.51)^{\mathrm{a}}\end{array}$ & $\begin{array}{c}46.67 \\
(43.36)^{\mathrm{e}}\end{array}$ & $\begin{array}{c}3.33 \\
(11.29)^{\mathrm{b}}\end{array}$ \\
\hline $\begin{array}{l}\text { Tridax procumbens } \\
\text { (leaf) }\end{array}$ & $\begin{array}{c}0.00 \\
(4.05)^{\mathrm{h}}\end{array}$ & $\begin{array}{c}3.33 \\
(10.52)^{\mathrm{f}}\end{array}$ & $\begin{array}{c}3.33 \\
(11.29)^{\mathrm{i}}\end{array}$ & $\begin{array}{c}6.67 \\
(15.52)^{g}\end{array}$ & $\begin{array}{c}6.67 \\
(15.52)^{\mathrm{f}}\end{array}$ & $\begin{array}{c}16.67 \\
(24.47)^{\mathrm{c}}\end{array}$ & $\begin{array}{c}76.67 \\
(61.43)^{\mathrm{b}}\end{array}$ & $\begin{array}{c}0.00 \\
(4.05)^{\mathrm{c}}\end{array}$ \\
\hline $\begin{array}{l}\text { Tagetes tenuifolia } \\
\text { (Flower) }\end{array}$ & $\begin{array}{c}3.33 \\
(10.52)^{\mathrm{g}}\end{array}$ & $\begin{array}{c}3.33 \\
(10.52)^{\mathrm{f}}\end{array}$ & $\begin{array}{c}10.00 \\
(18.90)^{\mathrm{gh}}\end{array}$ & $\begin{array}{c}13.33 \\
(21.83)^{\mathrm{f}}\end{array}$ & $\begin{array}{c}13.33 \\
(21.83)^{\mathrm{e}}\end{array}$ & $\begin{array}{c}26.67 \\
(31.40)^{\mathrm{a}}\end{array}$ & $\begin{array}{c}60.00 \\
(51.04)^{\mathrm{d}}\end{array}$ & $\begin{array}{c}0.00 \\
(4.05)^{\mathrm{c}}\end{array}$ \\
\hline NSKE (5\%) & $\begin{array}{c}0.00 \\
(4.05)^{\mathrm{h}}\end{array}$ & $\begin{array}{c}26.67 \\
(31.08)^{\mathrm{b}}\end{array}$ & $\begin{array}{c}40.00 \\
(39.51)^{\mathrm{c}}\end{array}$ & $\begin{array}{c}46.67 \\
(43.36)^{\mathrm{b}}\end{array}$ & $\begin{array}{c}53.33 \\
(47.18)^{\mathrm{b}}\end{array}$ & $\begin{array}{c}0.00 \\
(4.05)^{\mathrm{f}}\end{array}$ & $\begin{array}{c}46.67 \\
(43.36)^{\mathrm{e}}\end{array}$ & $\begin{array}{c}0.00 \\
(4.05)^{\mathrm{c}}\end{array}$ \\
\hline Untreated check & $\begin{array}{c}0.00 \\
(4.05)^{\mathrm{h}}\end{array}$ & $\begin{array}{c}0.00 \\
(4.05)^{\mathrm{g}}\end{array}$ & $\begin{array}{c}0.00 \\
(4.05)^{\mathrm{j}}\end{array}$ & $\begin{array}{c}0.00 \\
(4.05)^{\mathrm{h}}\end{array}$ & $\begin{array}{c}0.00 \\
(4.05)^{\mathrm{g}}\end{array}$ & $\begin{array}{c}0.00 \\
(4.05)^{\mathrm{f}}\end{array}$ & $\begin{array}{l}100.00 \\
(89.96)^{\mathrm{a}}\end{array}$ & $\begin{array}{c}0.00 \\
(4.05)^{\mathrm{c}}\end{array}$ \\
\hline $\begin{array}{l}\mathrm{SE} \\
\mathrm{CD}(0.05)\end{array}$ & $\begin{array}{l}1.04 \\
2.13\end{array}$ & $\begin{array}{l}1.67 \\
3.41\end{array}$ & $\begin{array}{l}1.84 \\
3.76\end{array}$ & $\begin{array}{l}2.22 \\
4.54\end{array}$ & $\begin{array}{l}2.25 \\
4.59\end{array}$ & $\begin{array}{l}1.34 \\
2.75\end{array}$ & $\begin{array}{l}3.37 \\
6.90\end{array}$ & $\begin{array}{l}0.39 \\
0.81\end{array}$ \\
\hline
\end{tabular}


Table.2 Effect of aqueous plant extracts (10\%) belonging to the Family, Fabaceae on the growth and development of the diamondback moth, P. xylostella

\begin{tabular}{|c|c|c|c|c|c|c|c|c|}
\hline \multirow[b]{2}{*}{ Treatments } & \multicolumn{5}{|c|}{ Larval mortality } & \multirow{2}{*}{$\begin{array}{c}\text { Pupal mortality } \\
(\%)\end{array}$} & \multicolumn{2}{|c|}{ Adult emergence (\%) } \\
\hline & 1DAT & 2DAT & 3DAT & 4DAT & 5DAT & & Normal & Malformed \\
\hline Cassia alata(leaf) & $\begin{array}{c}0.00 \\
(4.05)^{\mathrm{e}} \\
\end{array}$ & $\begin{array}{c}6.67 \\
(15.52)^{\mathrm{c}} \\
\end{array}$ & $\begin{array}{l}13.33 \\
(21.8)^{\mathrm{b}} \\
\end{array}$ & $\begin{array}{c}13.33 \\
(21.41)^{\mathrm{d}} \\
\end{array}$ & $\begin{array}{l}13.33 \\
(21.4)^{\mathrm{d}} \\
\end{array}$ & $\begin{array}{c}20.00 \\
(26.83)^{\mathrm{cd}} \\
\end{array}$ & $\begin{array}{r}66.67 \\
(54.71)^{\mathrm{c}} \\
\end{array}$ & $\begin{array}{c}0.00 \\
(4.05)^{\mathrm{c}}\end{array}$ \\
\hline $\begin{array}{l}\text { Cassia fistula } \\
\text { (leaf) }\end{array}$ & $\begin{array}{c}0.00 \\
(4.05)^{\mathrm{e}} \\
\end{array}$ & $\begin{array}{c}0.00 \\
(4.05)^{\mathrm{d}} \\
\end{array}$ & $\begin{array}{l}6.67 \\
(15.5)^{\mathrm{c}} \\
\end{array}$ & $\begin{array}{c}6.67 \\
(14.96)^{\mathrm{d}} \\
\end{array}$ & $\begin{array}{c}6.67 \\
(15.0)^{\mathrm{d}} \\
\end{array}$ & $\begin{array}{l}10.00 \\
(18.90)^{\mathrm{f}}\end{array}$ & $\begin{array}{c}80.00 \\
(63.41)^{\mathrm{b}} \\
\end{array}$ & $\begin{array}{c}3.33 \\
(11.29)^{\mathrm{b}} \\
\end{array}$ \\
\hline $\begin{array}{l}\text { Delonix regia } \\
\text { (leaf) }\end{array}$ & $\begin{array}{c}0.00 \\
(4.05)^{\mathrm{e}}\end{array}$ & $\begin{array}{c}3.33 \\
(11.29)^{\mathrm{c}}\end{array}$ & $\begin{array}{c}6.67 \\
(15.5)^{\mathrm{c}}\end{array}$ & $\begin{array}{c}6.67 \\
(14.96)^{\mathrm{d}}\end{array}$ & $\begin{array}{c}6.67 \\
(15.0)^{\mathrm{d}}\end{array}$ & $\begin{array}{c}16.67 \\
(24.47)^{\mathrm{de}}\end{array}$ & $\begin{array}{c}66.67 \\
(54.71)^{\mathrm{c}}\end{array}$ & $\begin{array}{c}10.00 \\
(18.43)^{\mathrm{a}}\end{array}$ \\
\hline $\begin{array}{l}\text { Gliricidia sepium } \\
\text { (leaf) }\end{array}$ & $\begin{array}{c}0.00 \\
(4.05)^{\mathrm{e}}\end{array}$ & $\begin{array}{c}3.33 \\
(11.29)^{\mathrm{c}}\end{array}$ & $\begin{array}{c}6.67 \\
(15.5)^{\mathrm{c}}\end{array}$ & $\begin{array}{c}6.67 \\
(14.96)^{\mathrm{d}}\end{array}$ & $\begin{array}{c}10.00 \\
(18.43)^{\mathrm{d}}\end{array}$ & $\begin{array}{c}50.00 \\
(45.27)^{\mathrm{a}} \\
\end{array}$ & $\begin{array}{c}40.00 \\
(39.22)^{\mathrm{d}}\end{array}$ & $\begin{array}{r}0.00 \\
(4.05)^{\mathrm{c}}\end{array}$ \\
\hline Leucaena leucocephala(leaf) & $\begin{array}{c}3.33 \\
(11.29)^{\mathrm{d}}\end{array}$ & $\begin{array}{c}13.33 \\
(21.83)^{\mathrm{b}} \\
\end{array}$ & $\begin{array}{l}20.00 \\
(26.9)^{\mathrm{b}}\end{array}$ & $\begin{array}{c}20.00 \\
(26.55)^{\mathrm{c}}\end{array}$ & $\begin{array}{l}33.33 \\
(35.3)^{\mathrm{c}}\end{array}$ & $\begin{array}{c}26.67 \\
(31.40)^{\mathrm{b}}\end{array}$ & $\begin{array}{c}40.00 \\
(39.22)^{\mathrm{d}}\end{array}$ & $\begin{array}{c}0.00 \\
(4.05)^{\mathrm{c}}\end{array}$ \\
\hline $\begin{array}{l}\text { Prosopis juliflora } \\
\text { (leaf) }\end{array}$ & $\begin{array}{c}53.33 \\
(47.18)^{\mathrm{a}} \\
\end{array}$ & $\begin{array}{c}76.67 \\
(61.43)^{\mathrm{a}} \\
\end{array}$ & $\begin{array}{l}80.00 \\
(63.8)^{\mathrm{a}} \\
\end{array}$ & $\begin{array}{c}93.33 \\
(75.01)^{\mathrm{a}} \\
\end{array}$ & $\begin{array}{c}96.67 \\
(79.5)^{\mathrm{a}}\end{array}$ & $\begin{array}{c}3.33 \\
(11.29)^{\mathrm{g}}\end{array}$ & $\begin{array}{c}0.00 \\
(4.05)^{\mathrm{f}}\end{array}$ & $\begin{array}{c}0.00 \\
(4.05)^{\mathrm{c}}\end{array}$ \\
\hline $\begin{array}{l}\text { Senna auriculata } \\
\text { (leaf) }\end{array}$ & $\begin{array}{c}0.00 \\
(4.05)^{\mathrm{e}}\end{array}$ & $\begin{array}{c}3.33 \\
(11.29)^{\mathrm{c}}\end{array}$ & $\begin{array}{c}3.33 \\
(11.3)^{\mathrm{c}}\end{array}$ & $\begin{array}{c}6.67 \\
(14.96)^{\mathrm{d}}\end{array}$ & $\begin{array}{c}6.67 \\
(15.0)^{\mathrm{d}}\end{array}$ & $\begin{array}{c}23.33 \\
(29.21)^{\mathrm{bc}}\end{array}$ & $\begin{array}{c}70.00 \\
(56.77)^{\mathrm{c}}\end{array}$ & $\begin{array}{c}0.00 \\
(4.05)^{\mathrm{c}}\end{array}$ \\
\hline $\begin{array}{l}\text { Sesbania grandiflora } \\
\text { (leaf) }\end{array}$ & $\begin{array}{c}16.67 \\
(24.47)^{\mathrm{b}}\end{array}$ & $\begin{array}{c}73.33 \\
(59.21)^{\mathrm{a}} \\
\end{array}$ & $\begin{array}{l}73.33 \\
(59.2)^{\mathrm{a}}\end{array}$ & $\begin{array}{c}73.33 \\
(58.89)^{\mathrm{b}} \\
\end{array}$ & $\begin{array}{r}73.33 \\
(58.9)^{\mathrm{b}}\end{array}$ & $\begin{array}{c}13.33 \\
(21.83)^{\mathrm{ef}}\end{array}$ & $\begin{array}{c}13.33 \\
(21.41)^{\mathrm{e}} \\
\end{array}$ & $\begin{array}{c}0.00 \\
(4.05)^{\mathrm{c}}\end{array}$ \\
\hline $\begin{array}{l}\text { Tephrosia purpurea } \\
\text { (leaf) }\end{array}$ & $\begin{array}{c}6.67 \\
(15.52)^{\mathrm{c}}\end{array}$ & $\begin{array}{c}6.67 \\
(15.52)^{\mathrm{c}}\end{array}$ & $\begin{array}{c}6.67 \\
(15.5)^{\mathrm{c}}\end{array}$ & $\begin{array}{c}6.67 \\
(14.96)^{\mathrm{d}}\end{array}$ & $\begin{array}{c}6.67 \\
(15.0)^{\mathrm{d}} \\
\end{array}$ & $\begin{array}{c}20.00 \\
(26.91)^{\mathrm{cd}}\end{array}$ & $\begin{array}{c}73.33 \\
(58.89)^{\mathrm{b}}\end{array}$ & $\begin{array}{c}0.00 \\
(4.05)^{\mathrm{c}}\end{array}$ \\
\hline NSKE $(5 \%)$ & $\begin{array}{c}3.33 \\
(11.29)^{\mathrm{d}}\end{array}$ & $\begin{array}{c}13.33 \\
(21.83)^{\mathrm{b}}\end{array}$ & $\begin{array}{l}20.00 \\
(26.9)^{b}\end{array}$ & $\begin{array}{c}20.00 \\
(20.00)^{\mathrm{c}}\end{array}$ & $\begin{array}{c}33.3 \\
(35.3)^{\mathrm{c}}\end{array}$ & $\begin{array}{c}0.00 \\
(4.05)^{\mathrm{h}}\end{array}$ & $\begin{array}{c}66.67 \\
(54.71)^{\mathrm{c}}\end{array}$ & $\begin{array}{c}0.00 \\
(4.05)^{\mathrm{c}}\end{array}$ \\
\hline Untreated check & $\begin{array}{c}0.00 \\
(4.05)^{\mathrm{e}}\end{array}$ & $\begin{array}{c}0.00 \\
(4.05)^{\mathrm{d}}\end{array}$ & $\begin{array}{c}0.00 \\
(4.05)^{\mathrm{d}}\end{array}$ & $\begin{array}{c}0.00 \\
(4.05)^{\mathrm{e}}\end{array}$ & $\begin{array}{c}0.00 \\
(4.05)^{\mathrm{f}}\end{array}$ & $\begin{array}{c}0.00 \\
(4.05)^{\mathrm{h}}\end{array}$ & $\begin{array}{c}100 \\
(89.96)^{\mathrm{a}}\end{array}$ & $\begin{array}{c}0.00 \\
(4.05)^{\mathrm{c}}\end{array}$ \\
\hline $\begin{array}{c}\mathrm{SE} \\
\mathrm{CD}(0.05)\end{array}$ & $\begin{array}{l}1.02 \\
2.13\end{array}$ & $\begin{array}{l}2.24 \\
4.65\end{array}$ & $\begin{array}{l}2.75 \\
570\end{array}$ & $\begin{array}{l}3.45 \\
7.16\end{array}$ & $\begin{array}{l}3.26 \\
6.77\end{array}$ & $\begin{array}{l}1.55 \\
3.22\end{array}$ & $\begin{array}{l}3.57 \\
7.42\end{array}$ & $\begin{array}{l}1.07 \\
2.22\end{array}$ \\
\hline
\end{tabular}

Table.3 Effect of aqueous plant extracts(10\%) belonging to the Family, Bignoniaceae,

Cyperaceae, Euphorbiaceae, Lamiaceae, Malvaceae, Meliaceae, Myrtaceae, Pedaliaceae,

Phyllanthaceae and Piperaceaeson the growth and development of the diamondbackmoth, $P$. xylostella

\begin{tabular}{|c|c|c|c|c|c|c|c|c|}
\hline \multirow[b]{2}{*}{ Treatments } & \multicolumn{5}{|c|}{ Larval mortality (\%) } & \multirow{2}{*}{$\begin{array}{c}\text { Pupal mortality } \\
(\%)\end{array}$} & \multicolumn{2}{|c|}{ Adult emergence (\%) } \\
\hline & 1DAT & 2DAT & 3DAT & 4DAT & 5DAT & & Normal & Malformed \\
\hline Spathodea campanulata (flower) & $\begin{array}{c}6.67 \\
(15.52)^{\mathrm{d}}\end{array}$ & $\begin{array}{c}13.33 \\
(21.83)^{\mathrm{d}}\end{array}$ & $\begin{array}{c}13.33 \\
(21.83)^{\mathrm{e}}\end{array}$ & $\begin{array}{c}13.33 \\
(21.83)^{\mathrm{e}}\end{array}$ & $\begin{array}{c}16.67 \\
(24.47)^{\mathrm{e}}\end{array}$ & $\begin{array}{c}16.67 \\
(24.47)^{\mathrm{e}}\end{array}$ & $\begin{array}{c}60.00 \\
(51.04)^{\mathrm{d}}\end{array}$ & $\begin{array}{c}6.67 \\
(15.52)^{\mathrm{b}}\end{array}$ \\
\hline $\begin{array}{l}\text { Cyperus rotundus } \\
\text { (leaf) }\end{array}$ & $\begin{array}{c}0.00 \\
(4.05)^{\mathrm{f}}\end{array}$ & $\begin{array}{c}0.00 \\
(4.05)^{g}\end{array}$ & $\begin{array}{c}6.67 \\
(15.52)^{g}\end{array}$ & $\begin{array}{c}6.67 \\
(15.52)^{\mathrm{f}}\end{array}$ & $\begin{array}{c}6.67 \\
(15.52)^{\mathrm{f}}\end{array}$ & $\begin{array}{c}53.33 \\
(47.18)^{\mathrm{a}}\end{array}$ & $\begin{array}{c}40.00 \\
(39.51)^{\mathrm{e}}\end{array}$ & $\begin{array}{c}0.00 \\
(4.05)^{\mathrm{d}}\end{array}$ \\
\hline $\begin{array}{l}\text { Jatropha curcas } \\
\text { (leaf) }\end{array}$ & $\begin{array}{c}13.33 \\
(21.83)^{\mathrm{c}}\end{array}$ & $\begin{array}{c}13.33 \\
(21.83)^{\mathrm{d}}\end{array}$ & $\begin{array}{c}30.00 \\
(33.51)^{\mathrm{c}}\end{array}$ & $\begin{array}{c}56.67 \\
(49.10)^{\mathrm{a}} \\
\end{array}$ & $\begin{array}{c}66.67 \\
(55.02)^{\mathrm{a}} \\
\end{array}$ & $\begin{array}{c}23.33 \\
(29.21)^{\mathrm{d}} \\
\end{array}$ & $\begin{array}{c}10.00 \\
(18.90)^{\mathrm{g}}\end{array}$ & $\begin{array}{c}0.00 \\
(4.05)^{\mathrm{d}}\end{array}$ \\
\hline $\begin{array}{l}\text { Ricinus communis } \\
\text { (leaf) }\end{array}$ & $\begin{array}{c}6.67 \\
(15.52)^{\mathrm{d}}\end{array}$ & $\begin{array}{c}13.33 \\
(21.83)^{\mathrm{d}}\end{array}$ & $\begin{array}{c}16.67 \\
(24.47)^{\mathrm{e}}\end{array}$ & $\begin{array}{c}36.67 \\
(37.55)^{\mathrm{c}}\end{array}$ & $\begin{array}{c}36.67 \\
(37.55)^{\mathrm{d}}\end{array}$ & $\begin{array}{c}30.00 \\
(33.51)^{\mathrm{c}}\end{array}$ & $\begin{array}{c}26.67 \\
(31.40)^{\mathrm{f}}\end{array}$ & $\begin{array}{c}6.67 \\
(15.52)^{\mathrm{b}}\end{array}$ \\
\hline $\begin{array}{l}\text { Ocimum tenuiflorum } \\
\text { (leaf) }\end{array}$ & $\begin{array}{c}6.67 \\
(15.52)^{\mathrm{d}}\end{array}$ & $\begin{array}{c}10.00 \\
(18.90)^{\mathrm{e}}\end{array}$ & $\begin{array}{c}10.00 \\
(18.90)^{\mathrm{f}}\end{array}$ & $\begin{array}{c}13.33 \\
(21.83)^{\mathrm{e}}\end{array}$ & $\begin{array}{c}13.33 \\
(21.83)^{\mathrm{e}}\end{array}$ & $\begin{array}{c}6.67 \\
(15.52)^{\mathrm{f}}\end{array}$ & $\begin{array}{c}76.67 \\
(61.43)^{\mathrm{c}}\end{array}$ & $\begin{array}{c}3.33 \\
(11.29)^{\mathrm{c}}\end{array}$ \\
\hline $\begin{array}{l}\text { Abutilon indicum } \\
\text { (leaf) }\end{array}$ & $\begin{array}{c}40.00 \\
(39.51)^{\mathrm{a}}\end{array}$ & $\begin{array}{c}46.67 \\
(43.36)^{\mathrm{a}}\end{array}$ & $\begin{array}{c}46.67 \\
(43.36)^{\mathrm{a}}\end{array}$ & $\begin{array}{c}53.33 \\
(47.18)^{\mathrm{b}}\end{array}$ & $\begin{array}{c}60.00 \\
(51.04)^{\mathrm{b}}\end{array}$ & $\begin{array}{c}6.67 \\
(15.52)^{\mathrm{f}}\end{array}$ & $\begin{array}{c}33.33 \\
(35.55)^{\text {ef }}\end{array}$ & $\begin{array}{c}0.00 \\
(4.05)^{\mathrm{d}}\end{array}$ \\
\hline $\begin{array}{l}\text { Azadirachta indica } \\
\text { (leaf) }\end{array}$ & $\begin{array}{c}6.67 \\
(15.52)^{\mathrm{d}}\end{array}$ & $\begin{array}{c}13.33 \\
(21.83)^{\mathrm{d}}\end{array}$ & $\begin{array}{c}13.33 \\
(21.83)^{\mathrm{e}}\end{array}$ & $\begin{array}{c}53.33 \\
(47.18)^{\mathrm{b}}\end{array}$ & $\begin{array}{c}53.33 \\
(47.18)^{\mathrm{bc}}\end{array}$ & $\begin{array}{c}46.67 \\
(43.36)^{\mathrm{a}}\end{array}$ & $\begin{array}{c}0.00 \\
(4.05)^{\mathrm{h}}\end{array}$ & $\begin{array}{c}0.00 \\
(4.05)^{\mathrm{d}}\end{array}$ \\
\hline $\begin{array}{l}\text { Melia azedarach } \\
\text { (leaf) }\end{array}$ & $\begin{array}{c}6.67 \\
(15.52)^{\mathrm{d}}\end{array}$ & $\begin{array}{c}6.67 \\
(15.52)^{\mathrm{f}}\end{array}$ & $\begin{array}{c}6.67 \\
(15.52)^{\mathrm{g}}\end{array}$ & $\begin{array}{c}6.67 \\
(15.52)^{\mathrm{f}}\end{array}$ & $\begin{array}{c}13.33 \\
(21.83)^{\mathrm{e}}\end{array}$ & $\begin{array}{c}36.67 \\
(37.54)^{\mathrm{b}}\end{array}$ & $\begin{array}{c}40.00 \\
(39.50)^{\mathrm{e}}\end{array}$ & $\begin{array}{c}10.00 \\
(18.9)^{\mathrm{a}}\end{array}$ \\
\hline $\begin{array}{l}\text { Eucalyptus deglupta } \\
\text { (leaf) }\end{array}$ & $\begin{array}{c}23.33 \\
(29.21)^{\mathrm{b}}\end{array}$ & $\begin{array}{r}26.67 \\
(31.40)^{\mathrm{c}}\end{array}$ & $\begin{array}{c}23.33 \\
(29.21)^{\mathrm{d}}\end{array}$ & $\begin{array}{c}26.67 \\
(31.40)^{\mathrm{d}}\end{array}$ & $\begin{array}{c}36.67 \\
(37.55)^{\mathrm{d}}\end{array}$ & $\begin{array}{c}30.00 \\
(33.50)^{\mathrm{c}}\end{array}$ & $\begin{array}{c}33.33 \\
(35.55)^{\mathrm{e}}\end{array}$ & $\begin{array}{c}0.00 \\
(4.05)^{\mathrm{d}}\end{array}$ \\
\hline $\begin{array}{l}\text { Pedalium murex } \\
\text { (leaf) }\end{array}$ & $\begin{array}{c}0.00 \\
(4.05)^{\mathrm{f}}\end{array}$ & $\begin{array}{c}0.00 \\
(4.05)^{\mathrm{g}}\end{array}$ & $\begin{array}{c}6.67 \\
(15.52)^{\mathrm{g}}\end{array}$ & $\begin{array}{c}6.67 \\
(15.52)^{\mathrm{f}}\end{array}$ & $\begin{array}{c}6.67 \\
(15.52)^{\mathrm{f}}\end{array}$ & $\begin{array}{c}6.67 \\
(15.52)^{\mathrm{f}}\end{array}$ & $\begin{array}{c}86.67 \\
(68.98)^{\mathrm{b}}\end{array}$ & $\begin{array}{c}0.00 \\
(4.05)^{\mathrm{d}}\end{array}$ \\
\hline $\begin{array}{l}\text { Phylanthus niruri } \\
\text { (leaf) }\end{array}$ & $\begin{array}{c}40.00 \\
(39.51)^{\mathrm{a}}\end{array}$ & $\begin{array}{c}40.00 \\
(39.51)^{\mathrm{b}}\end{array}$ & $\begin{array}{c}46.67 \\
(43.36)^{\mathrm{a}}\end{array}$ & $\begin{array}{c}46.67 \\
(43.35)^{\mathrm{b}}\end{array}$ & $\begin{array}{c}46.67 \\
(43.36)^{\mathrm{c}}\end{array}$ & $\begin{array}{c}13.33 \\
(21.82)^{\mathrm{e}}\end{array}$ & $\begin{array}{c}40.00 \\
(39.50)^{\mathrm{e}}\end{array}$ & $\begin{array}{c}0.00 \\
(4.05)^{d}\end{array}$ \\
\hline $\begin{array}{l}\text { Piper nigrum } \\
\text { (leaf) }\end{array}$ & $\begin{array}{c}3.33 \\
(11.29)^{\mathrm{e}}\end{array}$ & $\begin{array}{c}6.67 \\
(15.52)^{\mathrm{f}}\end{array}$ & $\begin{array}{c}6.67 \\
(15.52)^{\mathrm{g}}\end{array}$ & $\begin{array}{c}6.67 \\
(15.52)^{\mathrm{f}}\end{array}$ & $\begin{array}{c}13.33 \\
(21.83)^{\mathrm{e}}\end{array}$ & $\begin{array}{c}26.67 \\
(31.40)^{\mathrm{c}}\end{array}$ & $\begin{array}{c}60.00 \\
(51.04)^{\mathrm{d}}\end{array}$ & $\begin{array}{c}0.00 \\
(4.05)^{\mathrm{d}}\end{array}$ \\
\hline $\begin{array}{l}\text { NSKE } \\
(5 \%)\end{array}$ & $\begin{array}{c}0.00 \\
(4.05)^{\mathrm{f}}\end{array}$ & $\begin{array}{c}10.00 \\
(18.90)^{\mathrm{e}}\end{array}$ & $\begin{array}{c}36.67 \\
(37.55)^{\mathrm{b}}\end{array}$ & $\begin{array}{c}46.67 \\
(43.35)^{\mathrm{b}}\end{array}$ & $\begin{array}{c}46.67 \\
(43.36)^{\mathrm{c}}\end{array}$ & $\begin{array}{c}13.33 \\
(21.82)^{\mathrm{e}}\end{array}$ & $\begin{array}{c}40.00 \\
(39.50)^{\mathrm{e}}\end{array}$ & $\begin{array}{c}0.00 \\
(4.05)^{\mathrm{d}}\end{array}$ \\
\hline Untreated check & $\begin{array}{c}0.00 \\
(4.05)^{\mathrm{f}}\end{array}$ & $\begin{array}{c}0.00 \\
(4.05)^{\mathrm{g}}\end{array}$ & $\begin{array}{c}0.00 \\
(4.05)^{\mathrm{h}}\end{array}$ & $\begin{array}{c}0.00 \\
(4.05)^{\mathrm{g}}\end{array}$ & $\begin{array}{c}0.00 \\
(4.05)^{\mathrm{h}}\end{array}$ & $\begin{array}{c}0.00 \\
(4.05)^{\mathrm{g}}\end{array}$ & $\begin{array}{c}100 \\
(89.96)^{\mathrm{a}}\end{array}$ & $\begin{array}{c}0.00 \\
(4.05)^{\mathrm{d}}\end{array}$ \\
\hline SE & 1.02 & 1.18 & 1.36 & 2.39 & 1.97 & 1.89 & 3.22 & 0.50 \\
\hline $\mathrm{CD}(0.05)$ & 2.10 & 2.42 & 2.79 & 4.89 & 4.03 & 3.87 & 6.61 & 1.03 \\
\hline
\end{tabular}


Table.4 Effect of aqueous extracts (10\%) belonging to the Family, Poaceae, Polygonaceae, Rutaceae, Sapindaceae, Solanaceae, Sterculiaceae, Verbenaceae and Zingiberaceae on the growth and development of the diamondback moth, $P$. xylostella

\begin{tabular}{|c|c|c|c|c|c|c|c|c|}
\hline \multirow[b]{2}{*}{ Treatments } & \multicolumn{5}{|c|}{ Larval mortality (\%) } & \multirow{2}{*}{$\begin{array}{c}\text { Pupal } \\
\text { mortality } \\
(\%)\end{array}$} & \multicolumn{2}{|c|}{ Adult emergence (\%) } \\
\hline & 1DAT & 2DAT & 3DAT & 4DAT & 5DAT & & Normal & Malformed \\
\hline $\begin{array}{l}\text { Andropogan muricatus } \\
\text { (leaf) }\end{array}$ & $\begin{array}{c}10.00 \\
(18.43)^{\mathrm{d}}\end{array}$ & $\begin{array}{c}6.67 \\
(15.52)^{b}\end{array}$ & $\begin{array}{c}6.67 \\
(14.96)^{\mathrm{d}}\end{array}$ & $\begin{array}{c}13.33 \\
(21.83)^{\mathrm{d}}\end{array}$ & $\begin{array}{c}13.33 \\
(21.41)^{\mathrm{c}}\end{array}$ & $\begin{array}{c}13.33 \\
(21.41)^{\mathrm{e}}\end{array}$ & $\begin{array}{c}66.67 \\
(55.02)^{\text {cde }}\end{array}$ & $\begin{array}{c}6.67 \\
(15.52)^{\mathrm{a}}\end{array}$ \\
\hline $\begin{array}{l}\text { Cymbopogon } \\
\text { schoenanthus (leaf) }\end{array}$ & $\begin{array}{c}0.00 \\
(4.05)^{\mathrm{e}}\end{array}$ & $\begin{array}{c}0.00 \\
(4.05)^{\mathrm{d}}\end{array}$ & $\begin{array}{c}0.00 \\
(4.05)^{\mathrm{e}}\end{array}$ & $\begin{array}{c}0.00 \\
(4.05)^{\mathrm{g}}\end{array}$ & $\begin{array}{c}3.33 \\
(10.52)^{\mathrm{f}}\end{array}$ & $\begin{array}{c}60.00 \\
(50.75)^{\mathrm{a}}\end{array}$ & $\begin{array}{c}30.00 \\
(33.51)^{\mathrm{h}}\end{array}$ & $\begin{array}{c}6.67 \\
(15.52)^{\mathrm{a}}\end{array}$ \\
\hline $\begin{array}{l}\text { Cynodon dactylon } \\
\text { (leaf) }\end{array}$ & $\begin{array}{c}0.00 \\
(4.05)^{\mathrm{e}}\end{array}$ & $\begin{array}{c}6.67 \\
(15.52)^{b}\end{array}$ & $\begin{array}{c}6.67 \\
(14.96)^{\mathrm{d}}\end{array}$ & $\begin{array}{c}6.67 \\
(15.52)^{\mathrm{f}}\end{array}$ & $\begin{array}{c}6.67 \\
(14.96)^{\mathrm{e}}\end{array}$ & $\begin{array}{c}26.67 \\
(31.08)^{\mathrm{bc}}\end{array}$ & $\begin{array}{c}66.67 \\
(55.02)^{\text {cde }}\end{array}$ & $\begin{array}{c}0.00 \\
(4.05)^{\mathrm{c}}\end{array}$ \\
\hline $\begin{array}{l}\text { Antigonon leptopus } \\
\text { (leaf) }\end{array}$ & $\begin{array}{c}0.00 \\
(4.05)^{\mathrm{e}}\end{array}$ & $\begin{array}{c}3.33 \\
(11.29)^{\mathrm{c}}\end{array}$ & $\begin{array}{c}3.33 \\
(10.52)^{\mathrm{d}}\end{array}$ & $\begin{array}{c}6.67 \\
(15.52)^{\mathrm{f}}\end{array}$ & $\begin{array}{c}6.67 \\
(14.96)^{\mathrm{e}}\end{array}$ & $\begin{array}{c}13.33 \\
(21.41)^{\mathrm{e}}\end{array}$ & $\begin{array}{c}73.33 \\
(59.21)^{\mathrm{cd}}\end{array}$ & $\begin{array}{c}6.67 \\
(15.52)^{\mathrm{a}}\end{array}$ \\
\hline $\begin{array}{l}\text { Murraya koenigii } \\
\text { (leaf) }\end{array}$ & $\begin{array}{c}20.00 \\
(26.55)^{\mathrm{a}}\end{array}$ & $\begin{array}{c}20.00 \\
(26.91)^{\mathrm{a}}\end{array}$ & $\begin{array}{c}26.67 \\
(31.08)^{b}\end{array}$ & $\begin{array}{c}26.67 \\
(31.40)^{\mathrm{b}}\end{array}$ & $\begin{array}{c}33.33 \\
(35.55)^{\mathrm{b}}\end{array}$ & $\begin{array}{c}13.33 \\
(21.41)^{\mathrm{e}}\end{array}$ & $\begin{array}{c}53.33 \\
(46.89)^{\mathrm{fg}}\end{array}$ & $\begin{array}{c}0.00 \\
(4.05)^{\mathrm{c}}\end{array}$ \\
\hline $\begin{array}{l}\text { Cardiospermum } \\
\text { halicacabum (leaf) }\end{array}$ & $\begin{array}{c}0.00 \\
(4.05)^{\mathrm{e}}\end{array}$ & $\begin{array}{c}0.00 \\
(4.05)^{\mathrm{d}}\end{array}$ & $\begin{array}{c}3.33 \\
(10.52)^{\mathrm{d}}\end{array}$ & $\begin{array}{c}6.67 \\
(15.52)^{\mathrm{f}}\end{array}$ & $\begin{array}{c}6.67 \\
(14.96)^{\mathrm{e}}\end{array}$ & $\begin{array}{c}13.33 \\
(21.41)^{\mathrm{e}}\end{array}$ & $\begin{array}{c}80.00 \\
(63.77)^{\mathrm{b}}\end{array}$ & $\begin{array}{c}0.00 \\
(4.05)^{\mathrm{c}}\end{array}$ \\
\hline $\begin{array}{l}\text { Datura metal } \\
\text { (leaf) }\end{array}$ & $\begin{array}{c}0.00 \\
(4.05)^{\mathrm{e}}\end{array}$ & $\begin{array}{c}3.33 \\
(11.29)^{\mathrm{c}}\end{array}$ & $\begin{array}{c}6.67 \\
(14.96)^{\mathrm{d}}\end{array}$ & $\begin{array}{c}10.00 \\
(18.90)^{\mathrm{e}}\end{array}$ & $\begin{array}{c}10.00 \\
(18.43)^{\mathrm{d}}\end{array}$ & $\begin{array}{c}26.67 \\
(31.08)^{\mathrm{bc}}\end{array}$ & $\begin{array}{c}63.33 \\
(53.01)^{\mathrm{de}}\end{array}$ & $\begin{array}{c}0.00 \\
(4.05)^{\mathrm{c}}\end{array}$ \\
\hline $\begin{array}{l}\text { Solanum virginianum } \\
\text { (Fruit) }\end{array}$ & $\begin{array}{c}13.33 \\
(21.41)^{\mathrm{c}}\end{array}$ & $\begin{array}{c}20.00 \\
(26.91)^{\mathrm{a}}\end{array}$ & $\begin{array}{c}20.00 \\
(26.55)^{\mathrm{b}}\end{array}$ & $\begin{array}{c}30.00 \\
(33.51)^{\mathrm{b}}\end{array}$ & $\begin{array}{c}30.00 \\
(33.20)^{\mathrm{b}}\end{array}$ & $\begin{array}{c}20.00 \\
(26.55)^{\mathrm{d}}\end{array}$ & $\begin{array}{c}50.00 \\
(45.27)^{\mathrm{g}}\end{array}$ & $\begin{array}{c}0.00 \\
(4.05)^{\mathrm{c}}\end{array}$ \\
\hline $\begin{array}{l}\text { Helictres isora } \\
\text { (Fruit) }\end{array}$ & $\begin{array}{c}0.00 \\
(4.05)^{\mathrm{e}}\end{array}$ & $\begin{array}{c}0.00 \\
(4.05)^{\mathrm{d}}\end{array}$ & $\begin{array}{c}16.67 \\
(24.09)^{\mathrm{c}}\end{array}$ & $\begin{array}{c}16.67 \\
(24.47)^{\mathrm{c}}\end{array}$ & $\begin{array}{c}16.67 \\
(24.09)^{\mathrm{c}}\end{array}$ & $\begin{array}{c}23.33 \\
(28.87)^{\mathrm{c}}\end{array}$ & $\begin{array}{c}56.67 \\
(49.10)^{\mathrm{efg}}\end{array}$ & $\begin{array}{c}3.33 \\
(11.29)^{\mathrm{b}}\end{array}$ \\
\hline $\begin{array}{l}\text { Lantana camara } \\
\text { (leaf) }\end{array}$ & $\begin{array}{c}0.00 \\
(4.05)^{\mathrm{e}}\end{array}$ & $\begin{array}{c}0.00 \\
(4.05)^{\mathrm{d}}\end{array}$ & $\begin{array}{c}3.33 \\
(10.52)^{\mathrm{d}}\end{array}$ & $\begin{array}{c}6.67 \\
(15.52)^{f}\end{array}$ & $\begin{array}{c}6.67 \\
(14.96)^{\mathrm{e}}\end{array}$ & $\begin{array}{c}13.33 \\
(21.41)^{\mathrm{e}}\end{array}$ & $\begin{array}{c}76.67 \\
(61.43)^{\mathrm{bc}}\end{array}$ & $\begin{array}{c}3.33 \\
(11.29)^{\mathrm{b}}\end{array}$ \\
\hline $\begin{array}{l}\text { Curcuma longa } \\
\text { (rhizome) }\end{array}$ & $\begin{array}{c}16.67 \\
(24.09)^{\mathrm{b}}\end{array}$ & $\begin{array}{c}23.33 \\
(29.21)^{\mathrm{a}}\end{array}$ & $\begin{array}{c}30.00 \\
(33.20)^{\mathrm{a}}\end{array}$ & $\begin{array}{c}53.33 \\
(47.18)^{\mathrm{a}}\end{array}$ & $\begin{array}{c}56.67 \\
(48.81)^{\mathrm{a}}\end{array}$ & $\begin{array}{c}30.00 \\
(33.20)^{\mathrm{b}}\end{array}$ & $\begin{array}{c}13.33 \\
(21.83)^{\mathrm{i}}\end{array}$ & $\begin{array}{c}0.00 \\
(4.05)^{\mathrm{c}}\end{array}$ \\
\hline $\begin{array}{l}\text { NSKE } \\
(5 \%)\end{array}$ & $\begin{array}{c}0.00 \\
(4.05)^{\mathrm{e}}\end{array}$ & $\begin{array}{c}20.00 \\
(26.91)^{\mathrm{a}}\end{array}$ & $\begin{array}{c}20.00 \\
(26.55)^{\mathrm{b}}\end{array}$ & $\begin{array}{c}26.67 \\
(31.40)^{\mathrm{b}}\end{array}$ & $\begin{array}{c}33.33 \\
(35.55)^{\mathrm{b}}\end{array}$ & $\begin{array}{c}13.33 \\
(21.41)^{\mathrm{e}}\end{array}$ & $\begin{array}{c}53.33 \\
(46.89)^{\mathrm{fg}}\end{array}$ & $\begin{array}{c}0.00 \\
(4.05)^{\mathrm{c}}\end{array}$ \\
\hline Untreated check & $\begin{array}{c}0.00 \\
(4.05)^{\mathrm{e}}\end{array}$ & $\begin{array}{c}0.00 \\
(4.05)^{\mathrm{e}}\end{array}$ & $\begin{array}{c}0.00 \\
(4.05)^{\mathrm{f}}\end{array}$ & $\begin{array}{c}0.00 \\
(4.05)^{\mathrm{h}}\end{array}$ & $\begin{array}{c}0.00 \\
(4.05)^{\mathrm{h}}\end{array}$ & $\begin{array}{c}0.00 \\
(4.05)^{\mathrm{f}}\end{array}$ & $\begin{array}{c}100.00 \\
(89.96)^{\mathrm{a}}\end{array}$ & $\begin{array}{c}0.00 \\
(4.05)^{\mathrm{c}}\end{array}$ \\
\hline $\begin{array}{l}\mathrm{SE} \\
\mathrm{CD}(0.05)\end{array}$ & $\begin{array}{l}0.65 \\
1.34\end{array}$ & $\begin{array}{l}1.40 \\
2.88\end{array}$ & $\begin{array}{l}2.77 \\
5.70\end{array}$ & $\begin{array}{l}1.25 \\
2.58\end{array}$ & $\begin{array}{l}1.30 \\
2.67\end{array}$ & $\begin{array}{l}1.81 \\
3.72\end{array}$ & $\begin{array}{l}3.65 \\
7.52\end{array}$ & $\begin{array}{l}0.53 \\
1.10\end{array}$ \\
\hline
\end{tabular}


Table.5 Effect of selected aqueous plant extracts (10\%) on the growth and development of the diamondback moth, P. xylostella

\begin{tabular}{|c|c|c|c|c|c|c|c|c|}
\hline \multirow{2}{*}{ Treatments } & \multicolumn{5}{|c|}{ Larval mortality (\%) } & \multirow{2}{*}{$\begin{array}{c}\text { Pupal } \\
\text { mortality }(\%)\end{array}$} & \multicolumn{2}{|c|}{ Adult emergence (\%) } \\
\hline & 1DAT & 2DAT & 3DAT & 4DAT & 5DAT & & Normal & Malformed \\
\hline $\begin{array}{l}\text { Andrographis } \\
\text { paniculata (leaf) }\end{array}$ & $\begin{array}{c}40.00 \\
(39.51)^{\mathrm{b}}\end{array}$ & $\begin{array}{c}66.67 \\
(55.02)^{\mathrm{a}}\end{array}$ & $\begin{array}{c}66.67 \\
(55.02)^{\mathrm{a}}\end{array}$ & $\begin{array}{c}73.33 \\
(59.21)^{\mathrm{b}}\end{array}$ & $\begin{array}{c}73.33 \\
(59.21)^{\mathrm{b}}\end{array}$ & $\begin{array}{c}26.67 \\
(31.40)^{\mathrm{a}}\end{array}$ & $\begin{array}{c}0.00 \\
(4.05)^{\mathrm{e}}\end{array}$ & $\begin{array}{c}0.00 \\
(4.05)^{\mathrm{b}}\end{array}$ \\
\hline $\begin{array}{l}\text { Jatropha curcas } \\
\text { (leaf) }\end{array}$ & $\begin{array}{c}10.00 \\
(18.90)^{\mathrm{c}}\end{array}$ & $\begin{array}{c}10.00 \\
(18.90)^{\mathrm{c}}\end{array}$ & $\begin{array}{c}26.67 \\
(31.40)^{\mathrm{c}}\end{array}$ & $\begin{array}{c}53.33 \\
(47.18)^{\mathrm{c}}\end{array}$ & $\begin{array}{c}63.33 \\
(53.01)^{\mathrm{b}}\end{array}$ & $\begin{array}{c}23.33 \\
(29.21)^{\mathrm{a}}\end{array}$ & $\begin{array}{c}10.00 \\
(18.90)^{\mathrm{d}}\end{array}$ & $\begin{array}{c}3.33 \\
(7.03)^{\mathrm{a}}\end{array}$ \\
\hline $\begin{array}{l}\text { Curcuma longa } \\
\text { (leaf) }\end{array}$ & $\begin{array}{c}13.33 \\
(21.83)^{\mathrm{c}}\end{array}$ & $\begin{array}{c}20.00 \\
(26.91)^{\mathrm{b}}\end{array}$ & $\begin{array}{c}26.67 \\
(31.40)^{\mathrm{c}}\end{array}$ & $\begin{array}{c}50.00 \\
(45.27)^{\mathrm{c}}\end{array}$ & $\begin{array}{c}53.33 \\
(47.18)^{\mathrm{c}}\end{array}$ & $\begin{array}{c}26.67 \\
(31.40)^{\mathrm{a}} \\
\end{array}$ & $\begin{array}{l}20.00 \\
(26.91)^{\mathrm{c}}\end{array}$ & $\begin{array}{c}0.00 \\
(4.05)^{\mathrm{b}}\end{array}$ \\
\hline $\begin{array}{l}\text { Prosopis juliflora } \\
\text { (leaf) }\end{array}$ & $\begin{array}{c}46.67 \\
(43.36)^{\mathrm{a}} \\
\end{array}$ & $\begin{array}{c}70.00 \\
(57.08)^{\mathrm{a}} \\
\end{array}$ & $\begin{array}{c}73.33 \\
(59.21)^{\mathrm{a}} \\
\end{array}$ & $\begin{array}{c}86.67 \\
(68.98)^{\mathrm{a}} \\
\end{array}$ & $\begin{array}{c}90.00 \\
(72.02)^{\mathrm{a}}\end{array}$ & $\begin{array}{c}10.00 \\
(18.90)^{\mathrm{c}}\end{array}$ & $\begin{array}{c}0.00 \\
(4.05)^{\mathrm{e}}\end{array}$ & $\begin{array}{c}0.00 \\
(4.05)^{\mathrm{b}}\end{array}$ \\
\hline $\begin{array}{l}\text { Sesbania grandiflora } \\
\text { (leaf) }\end{array}$ & $\begin{array}{c}6.67 \\
(15.52)^{\mathrm{d}}\end{array}$ & $\begin{array}{c}66.67 \\
(55.02)^{\mathrm{a}}\end{array}$ & $\begin{array}{c}66.67 \\
(55.02)^{\mathrm{b}}\end{array}$ & $\begin{array}{c}66.67 \\
(55.02)^{\mathrm{b}}\end{array}$ & $\begin{array}{c}66.67 \\
(55.02)^{\mathrm{c}}\end{array}$ & $\begin{array}{c}20.00 \\
(26.91)^{\mathrm{b}}\end{array}$ & $\begin{array}{c}13.33 \\
(21.83)^{\mathrm{c}}\end{array}$ & $\begin{array}{c}0.00 \\
(4.05)^{\mathrm{b}}\end{array}$ \\
\hline NSKE $(5 \%)$ & $\begin{array}{c}13.33 \\
(21.83)^{\mathrm{c}}\end{array}$ & $\begin{array}{c}20.00 \\
(26.91)^{\mathrm{b}}\end{array}$ & $\begin{array}{c}26.67 \\
(31.40)^{\mathrm{c}}\end{array}$ & $\begin{array}{c}50.00 \\
(45.27)^{\mathrm{c}}\end{array}$ & $\begin{array}{c}53.33 \\
(47.18)^{\mathrm{c}}\end{array}$ & $\begin{array}{c}0.00 \\
(4.05)^{\mathrm{d}}\end{array}$ & $\begin{array}{c}46.66 \\
(43.36)^{\mathrm{b}}\end{array}$ & $\begin{array}{c}0.00 \\
(4.05)^{\mathrm{b}}\end{array}$ \\
\hline Untreated check & $\begin{array}{c}0.00 \\
(4.05)^{\mathrm{e}}\end{array}$ & $\begin{array}{c}0.00 \\
(4.05)^{\mathrm{d}}\end{array}$ & $\begin{array}{c}0.00 \\
(4.05)^{\mathrm{d}}\end{array}$ & $\begin{array}{c}0.00 \\
(4.05)^{\mathrm{d}}\end{array}$ & $\begin{array}{c}0.00 \\
(4.05)^{\mathrm{d}}\end{array}$ & $\begin{array}{c}0.00 \\
(4.05)^{\mathrm{d}}\end{array}$ & $\begin{array}{l}100.00 \\
(89.96)^{\mathrm{a}}\end{array}$ & $\begin{array}{c}0.00 \\
(4.05)^{\mathrm{b}}\end{array}$ \\
\hline $\begin{array}{l}\mathrm{SE} \\
\mathrm{CD}(0.05)\end{array}$ & $\begin{array}{l}3.60 \\
1.68\end{array}$ & $\begin{array}{l}2.83 \\
6.07\end{array}$ & $\begin{array}{l}3.17 \\
6.80\end{array}$ & $\begin{array}{c}4.87 \\
10.44\end{array}$ & $\begin{array}{c}5.70 \\
12.23\end{array}$ & $\begin{array}{l}1.32 \\
2.84\end{array}$ & $\begin{array}{l}0.67 \\
1.44\end{array}$ & $\begin{array}{l}0.24 \\
0.52\end{array}$ \\
\hline
\end{tabular}

Table.6 Anti-feedant activity of selected aqueous plant extracts $(10 \%)$ on the diamondback moth, P. xylostella

\begin{tabular}{|c|c|c|c|c|}
\hline \multirow{3}{*}{ Treatments } & \multicolumn{2}{|c|}{ Leaf area fed $\left(\mathrm{cm}^{2}\right)$} & \multirow{2}{*}{\multicolumn{2}{|c|}{$\begin{array}{l}\text { Antifeedant } \\
\text { activity (\%) }\end{array}$}} \\
\hline & \multirow{2}{*}{1 DAT } & \multirow{2}{*}{ 2DAT } & & \\
\hline & & & 1 DAT & 2DAT \\
\hline $\begin{array}{l}\text { Andrographis paniculata } \\
\text { (leaf) }\end{array}$ & $\begin{array}{c}3.82 \\
(11.27)^{\mathrm{c}}\end{array}$ & $\begin{array}{c}5.32 \\
(13.33)^{\mathrm{c}}\end{array}$ & 46.64 & 50.83 \\
\hline $\begin{array}{l}\text { Jatropha curcas } \\
\text { (leaf) }\end{array}$ & $\begin{array}{c}5.66 \\
(13.76)^{b}\end{array}$ & $\begin{array}{c}7.16 \\
(15.51)^{b}\end{array}$ & 20.94 & 33.82 \\
\hline $\begin{array}{l}\text { Curcuma longa } \\
\text { (leaf) }\end{array}$ & $\begin{array}{c}6.16 \\
(14.36)^{b}\end{array}$ & $\begin{array}{c}8.32 \\
(16.76)^{b}\end{array}$ & 13.96 & 23.10 \\
\hline $\begin{array}{l}\text { Prosopis juliflora } \\
\text { (leaf) }\end{array}$ & $\begin{array}{c}4.00 \\
(11.53)^{\mathrm{c}}\end{array}$ & $\begin{array}{c}4.50 \\
(12.24)^{\mathrm{c}}\end{array}$ & 44.13 & 58.41 \\
\hline $\begin{array}{l}\text { Sesbania grandiflora } \\
\text { (leaf) }\end{array}$ & $\begin{array}{c}4.00 \\
(11.53)^{\mathrm{c}}\end{array}$ & $\begin{array}{c}5.16 \\
(13.12)^{\mathrm{c}}\end{array}$ & 44.13 & 52.31 \\
\hline $\operatorname{NSKE}(5 \%)$ & $\begin{array}{c}3.94 \\
(11.44)^{\mathrm{c}}\end{array}$ & $\begin{array}{c}5.34 \\
(13.36)^{\mathrm{c}}\end{array}$ & 44.97 & 50.64 \\
\hline Untreated check & $\begin{array}{c}7.16 \\
(15.51)^{\mathrm{a}} \\
\end{array}$ & $\begin{array}{c}10.82 \\
(19.20)^{\mathrm{a}}\end{array}$ & 0.00 & 0.00 \\
\hline $\begin{array}{l}\text { SE } \\
\mathrm{CD}(0.05)\end{array}$ & $\begin{array}{l}0.65 \\
1.41\end{array}$ & $\begin{array}{l}0.76 \\
1.64\end{array}$ & - & - \\
\hline
\end{tabular}


Table.7 Oviposition deterrent action of aqueous plant extracts (10\%) on the diamondback moth, P. xylostella by free choice test

\begin{tabular}{|l|c|c|}
\hline \multicolumn{1}{|c|}{ Treatments } & No. of eggs laid & Oviposition deterrence (\%) \\
\hline $\begin{array}{l}\text { Andrographis paniculata } \\
\text { (leaf) }\end{array}$ & $\begin{array}{c}15.00 \\
(3.87)^{\mathrm{c}}\end{array}$ & 62.81 \\
\hline $\begin{array}{l}\text { Jatropha curcas } \\
\text { (leaf) }\end{array}$ & $\begin{array}{c}15.00 \\
(3.87)^{\mathrm{c}}\end{array}$ & 62.81 \\
\hline $\begin{array}{l}\text { Curcuma longa } \\
\text { (leaf) }\end{array}$ & $\begin{array}{c}10.00 \\
(3.16)\end{array}$ & 75.21 \\
\hline $\begin{array}{l}\text { Prosopis juliflora } \\
\text { (leaf) }\end{array}$ & $\begin{array}{c}10.33 \\
(3.21)^{\mathrm{b}}\end{array}$ & 74.38 \\
\hline $\begin{array}{l}\text { Sesbania grandiflora } \\
\text { (leaf) }\end{array}$ & $\begin{array}{c}5.67 \\
(2.38)^{\mathrm{a}}\end{array}$ & 85.95 \\
\hline NSKE (5\%) & $\begin{array}{c}23.67 \\
(4.86)^{\mathrm{d}}\end{array}$ & 41.32 \\
\hline Untreated check & $\begin{array}{c}40.33 \\
(6.35)^{\mathrm{e}}\end{array}$ \\
\hline SE & 0.1266 & - \\
CD $(0.05)$ & 0.2717 & \\
\hline
\end{tabular}

Table.8 Oviposition deterrent action of aqueous plant extract (10\%) on the diamondback moth, P. xylostella by no choice test

\begin{tabular}{|l|c|c|}
\hline \multicolumn{1}{|c|}{ Treatments } & No. of eggs laid & Oviposition deterrence (\%) \\
\hline $\begin{array}{l}\text { Andrographis paniculata } \\
\text { (leaf) }\end{array}$ & $\begin{array}{c}25.67 \\
(5.06)^{\mathrm{c}}\end{array}$ & 30.63 \\
\hline $\begin{array}{l}\text { Jatropha curcas } \\
\text { (leaf) }\end{array}$ & $\begin{array}{c}22.00 \\
(4.69)^{\mathrm{b}}\end{array}$ & 40.54 \\
\hline $\begin{array}{l}\text { Curcuma longa } \\
\text { (leaf) }\end{array}$ & $\begin{array}{c}16.67 \\
(4.08)^{\mathrm{a}}\end{array}$ & 54.95 \\
\hline $\begin{array}{l}\text { Prosopis juliflora } \\
\text { (leaf) }\end{array}$ & $\begin{array}{c}14.33 \\
(3.78)^{\mathrm{a}}\end{array}$ & 61.26 \\
\hline $\begin{array}{l}\text { Sesbania grandiflora } \\
\text { (leaf) }\end{array}$ & $\begin{array}{c}16.00 \\
(4.00)^{\mathrm{a}}\end{array}$ \\
\hline NSKE (5\%) & $\begin{array}{c}22.33 \\
(4.72)^{\mathrm{b}}\end{array}$ & 56.76 \\
\hline Untreated check & $\begin{array}{c}37.00 \\
(6.08)^{\mathrm{d}}\end{array}$ \\
\hline SE & $\begin{array}{l}0.1472 \\
0.3158\end{array}$ \\
CD $(0.05)$ & & \\
\hline
\end{tabular}

Effect of selected plant products on the larval mortality, pupal mortality and adult emergence

Among the five botanical extracts tested at 10 per cent, maximum larval mortality was recorded on Andrographis paniculata (40.00 $\%)$ on 1 DAT. Highest mortality recorded on 5 DAT was in P. juliflora $(90.00 \%)$ followed by A. paniculata $(73.33 \%)$, S. grandiflora (66.67 \%), J. curcas (63.33\%) and C. longa $(53.33 \%)$. The pupal mortality was highest in A. paniculata (26.67 \%) which was statistically on par with $C$. longa (26.66 \%), J. curcas $(23.33 \%)$. No adult emergence was recorded in $P$. juliflora and $A$. paniculata. The malformed adult was observed in $J$. curcas (3.33\%) (Table 5). 
Torres et al., (2001) also reported that $P$. juliflora leaf extract was promising with 66.7 per cent larval mortality of $P$. xylostella. $P$. juliflora was also found to cause 13.33 per cent larval mortality of Spodoptera litura (Nelson and Venugopal, 2006). Chandrashekharaiah et al., (2015) also reported that the aqueous leaf extract of A.squamosa@10 per cent caused 66.70 per cent larval mortality in $P$. xylostella.

Dadang et al., (2001) also recorded 16.7 per cent pupal mortality of $P$. xylostella. The higher pupal mortality caused by $C$. rotundus might be due to the presence of $\alpha$-Cyperone that interfered with the adult emergence due to their growth regulating effects in insects which was documented in P. xylostella (Koul, 2009). The toxicity of $T$. tenuifolia and $A$. calamus to Spodoptera litura pupa was already reported by Nelson and Venugopal (2006).

Death in the pupal stage is ascribed to the slow action of plant products on growth stages of insect or due to the enhanced activity of plant constituents when assimilated in insect tissues.

Similar effects were documented by Senthamizhselvan and Muthukrishnan (1992) against Spodoptera exigua and Valler and Capinera (1993) against S. eridania.

Malformation of adults was observed in $S$. litura with extracts of Trianthema portulacastrum (3.33\%), Agave americana (6.67 \%), Ipomoea carnea (6.67 \%), Curcumis trigonus leaf (3.33\%), C. trigonus stem $(6.67 \%), C$. trigonus fruit $(16.67 \%)$, Allium sativum (6.67\%), Anamirta cocculus (10.00\%), Anethum graveolans (3.33\%), Vitex negundo (10.00\%), C. longa (3.33\%), Tribulus terrestris leaf $(6.67 \%)$ and $T$. terrestris fruit $(10.00 \%)$ (Nelson and Venugopal, 2006).
Anti-feedant activity of plant extracts on $\boldsymbol{P}$. xylostella

The leaf area fed by larvae was recorded to assess the anti-feedant activity of different plant extracts. On 1 DAT the leaf area fed was lowest in A. paniculata $(3.82 \mathrm{~cm} 2)$ which was on par with $P$. juliflora $(4.00 \mathrm{~cm} 2), S$. grandiflora (4.00 cm2) and NSKE (5\%) (3.94 $\mathrm{cm} 2)$. On 2 DAT the leaf area fed was lowest on $P$. juliflora $(4.56 \mathrm{~cm} 2)$. On 1 DAT, antifeedant activity was highest in $A$. paniculata (46.64 \%) followed by $P$. juliflora and $S$. grandiflora $(44.13 \%)$. On 2 DAT, maximum anti- feedant activity was observed in $P$. juliflora $(58.41 \%)$ followed by $S$. grandiflora $(52.31 \%)$ and A. paniculata $(50.83 \%$ ) (Table 6). Hermawan et al., (1994) who recorded lower leaf area consumed in leaves treated with A. paniculata $(4.32 \mathrm{~cm} 2)$.

Oviposition deterrent activity of plant extracts on $P$. xylostella

\section{Free choice test}

In free choice test, the number of eggs laid ranged from 5.67 to 15.00 in the plant extract sprayed plants as against 40.33 in the untreated control (Table 7). The oviposition deterrence was highest in $S$. grandiflora $(85.95 \%)$ which was significantly higher than all other plant extracts for $P$. xylostella. It was followed by $C$. longa $(75.21 \%)$ and $P$. juliflora $(74.38$ 6\%). The susceptible check, NSKE (5 \%) recorded an oviposition deterrent of 41.32 per cent.

\section{No choice test}

The number of eggs laid by $P$. xylostella in no choice test is presented in Table 8. Less number of eggs was laid in $P$. juliflora extract treated plants (14.33) followed by $S$. grandiflora (16.00) and C. longa (16.67) which were statistically on par. The 
oviposition deterrence percentage was found to be the highest in $P$. juliflora $(61.26 \%)$ followed by $S$. grandiflora $(56.76 \%)$ and $C$. longa $(54.95 \%)$.

Strong ovipositional deterrence by melon fruit fly has been observed when $5 \%$ neem oil and $5 \%$ alcohol extract of rambans were used. (Tomar et al., 2001). Hermawan et al., (1994) observed the anti-oviposition activities of the fractions of extract from a tropical plant, $A$. paniculata against Diamondback moth, $P$. xylostella.

\section{Acknowledgement}

The authors are grateful to the Department of Entomology, Tamil Nadu Agricultural University for providing facitilites to conduct this research.

\section{References}

Chandrashekharaiah, M. and V.T. Sannaveerappanavar. 2013. Biological activity of select plant and indigenous extracts against diamondback moth, Plutella xyllostella (L.) (Lepidoptera: Plutellidae) and cowpea aphid, Aphis craccivora Koch (Hemiptera: Aphididae). Current Biotica, 7(3): 134-144.

Chandrashekharaiah, M., S.C. Satish., K. Vasudev., V. Arya and G.M. Narasimhamurthy. 2015. Efficacy of plant and aboriginal preparations against diamondback moth, Plutella xylostella (L.) (Lepidoptera: Plutellidae). J. Entomol. Zool. Stud., 3(4): 18-23.

Chawla, R.P. and B.S. Joia. 1992. Studies on the development of resistance in the diamondback moth, Plutella xylostella (L.) to quinalphos in Punjab. J. Insect Sci. 5: 106-108.

Chawla, R.P. and R.L. Kalra. 1976. Studies on insecticide resistance in Plutella xylostella Linn. (diamondback moth). Indian J. Plant. Protect., 4: 170-179.

Dadang, E.D. Fitriasari and D. Prijono. 2011. Field efficacy of two botanical insecticide formulations against cabbage insect pests, Crocidolomia pavonana (F.) (Lepidoptera: Pyralidae) and Plutella xylostella(L.) (Lepidoptera: Yponomeutidae). J.ISSAAS., 17(2): 38-47.

Deshmukh, S.N. and P.U. Saramma. 1973. Comparative susceptibility of Plutella maculipennis (Curtis) collected from Ludhiana and Jalandhar districts to some insecticides. Pesticides, 7(1): 21-22.

Fletcher, T.B. 1914. Some South Indian Insects. Superintendent Government Press, Madras, $565 \mathrm{p}$.

Hermawan, W., S. Kajiyama., R. Tsukuda., K. Fujisaki., A. Kobayashi and F. Nakasuji. 1994. Antifeedant and antioviposition activities of the fractions of extract from atropical plant, Andrographis paniculata (Acanthaceae), against the diamondback moth, Plutella xylostella (Lepidoptera: Yponomeutidae). Appl. Entomol. Zool., 29: 533-538.

Isman, M. B. 2008. Botanical insecticides: For richer, for poorer. Pest Manag. Sci., 64: 811.

Mohan, M. and G.T. Gujar. 2003. Local variation in susceptibility of the diamondback moth, Plutella xylostella (L.) to insecticides and role of detoxification enzymes. Crop. Prot., 22(3): 495-504.

Nelson, S.J. and M.S.Venugopal. 2006. Antifeedant and growth disruptive effects of various plant products on Spodoptera litura K. (Lepidoptera: Noctuidae). J. ento Res., 30(2): 1-10.

NHB. 2017. Horticultural Statistics at a Glance 2015. National Horticulture Board, Ministry of Agriculture, GOI. 148 pp.

Oyedokun, A. V., Anikwe, J. C., Okelana, F. A., Mokwunye I. U. and Azeez O. M. 2011. Pesticidal efficacy of three tropical herbal plants' leaves extracts against Macrotermes bellicosus, an emerging pest of cocoa, Theobroma cacao L. Journal of Biopesticides, 4(2): 131-137.

$\mathrm{Pu}, \mathrm{X}$., Y.Yang and Y.Wu. 2010. Characterization of abamectin resistance in a field evolved multiresistant population of Plutella xylostella. Pest Manag. Sci., 66: 371-378.

Saxena, J.D., S. Rai., K.M. Srivastava and S.R. Sinha. 1989. Resistance in the field 
population of the diamondback moth to some commonly used synthetic pyrethroids. Indian J. Entomol., 51: 265-268.

Sayyed, A.H and D.J. Wright. 2006. Genetics and evidence for an esterase-associated mechanism of resistance to indoxacarb in a field population of diamondback moth (Lepidoptera: Plutellidae). Pest Manag. Sci., 62: 1045-1051.

Senthamizhselvan, M. and J. Muthukrishanan. 1992. Effect of plant chemicals on food consumption of three lepidopteran larvae. Insect Sci. Applic., 13 : 429-434.

Sharma,S., A. Senrung and A.K. Singh. 2014. Toxic effect of Neem, Azadirachtaindica (A. Juss) foliage extracts against Diamondback moth (DBM), Plutellaxylostella (L.) (Lepidoptera, Plutellidae). J. Biopest., 5(1):99-105.

Shelton, A. M., Hatch, S. L., Zhao, J. Z., Chen, M., Earle, E. D. and Cao, J. 2008. Suppression of diamondback moth using Bt-transfergenic plants as a trap crop. Crop Protection, 27: 403-409.

Singh J, A. K. Upadhyay., A. Bahadur., B. Singh and K.P. Singh. 2006. Antioxidant phytochemicals in cabbage (Brassica oleracea L. var. capitata). Scientia Horticulturae., 108: 233-237

Tabashnik, B.E. and N.L. Cushing. 1987. Leaf residue vs. topical bioassays for assessing insecticide resistance in diamond back moth, Plutella xylostella L. FAO Plant Prot. Bull., 35:11-14.

Tomar, P. and S. Gajendra. 2001. Efficacy of neem, certain plant extracts and oils as ovipositional deterrents against melon fruit fly, Bactrocera cucurbitae (Coq.). Proc.
NCCP, Udaipur, p. 39.

Torres, A., V.Jose and D. Oliveira. 2001. Effects of plant aqueous extracts on the development of Plutella xylostella (L.) (Lepidoptera: Plutellidae). Neotrop. Entomol., 30(1) : 151-156.

Valler, S.M. and J.L.Capinera. 1993. Response of larvae of the southern army worm Spodoptera eridania (Cramer) Lepidoptera: Noctuidae to selected botanical insecticides and soap. J.Agric.Ent., 10(3) : 145-153.

Verma, A. N. and G. S. Sandhu. 1968. Chemical control of diamondback moth, Plutella xylostella (Curtis). J. Res. Punjab Agric. Univ., 5: 420-423.

Villalobos M. J. and A. Robledo. 1998. Screening for anti-insect activity in Mediterranean plants. Ind. Crop Prod., 8: 183-194.

Wagh, V.D., K. V. Wagh., Y. N. Tandale and S. A. Salve. 2009. Phytochemical, pharmacological and phytopharmaceutics aspects of Sesbania grandiflora (Hadga). $J$. Pharm. Res., 2(5): 889-892.

Zalucki, M.P., A. Shabbir., D. Adamson and L. Shu-Sheng. 2012. Estimating the economic cost of one of the world's major insect pests, Plutella xylostella (Lepidoptera; Plutellidae): just how long is a piece of string. J. Econ Entomol., 105: 1115-1129.

Zhao, J.Z., H.L. Collins., Y.X. Li., R.F.L. Mau., G.D. Thompson., M. Hertlen., J.T. Andalono., R. Boykin and A.M. Shelton. 2006. Monitoring of diamondback moth (Lepidoptera: Plutellidae) resistance to spinosad, indoxacarb and emamectin benzoate. J. Econ. Entomol, 99: 176-186.

\section{How to cite this article:}

Sangavi, R. and Johnson Thangaraj Edward, Y.S. 2017. Anti-Insect Activities of Plant Extracts on the Diamondback Moth, Plutella xylostella (L.). Int.J.Curr.Microbiol.App.Sci. 6(12): 28-39. doi: https://doi.org/10.20546/ijcmas.2017.612.004 